\title{
Knockdown of circular RNA hsa_circ_0003204 inhibits oxidative stress and apoptosis through the miR-330-5p/Nod2 axis to ameliorate endothelial cell injury induced by low-density lipoprotein
}

\author{
BIN ZHANG ${ }^{I}$, YUFAN ZHANG ${ }^{1}, R U I L I^{I}, Y A N L I^{I}, W E I$ YAN ${ }^{2}$ \\ 'Department of Neurology, Yulin First Hospital, Yulin, Shaanxi, China \\ ${ }^{2}$ Department of Vasculocardiology, People's Hospital of Tongchuan, Tongchuan, Shaanxi, China
}

\begin{abstract}
Introduction: Atherosclerosis (AS) is the leading cause of cardiovascular disease. Circular RNA hsa_circ_0003204 (hsa_circ_0003204) was elevated in oxidized low-density lipoprotein (ox-LDL)induced human umbilical vein endothelial cells. However, the role and molecular mechanism of hsa_circ_0003204 in the AS process have not been studied.

Material and methods: Human primary aortic endothelial cells (HAECs) were treated with low-density lipoprotein (ox-LDL) to establish the AS model. The viability of ox-LDL-induced HAECs was assessed by counting kit-8 (CCK8) assay. Reactive oxygen species (ROS), malondialdehyde (MDA) and superoxide dismutase (SOD) levels in ox-LDL-induced HAECs supernatant were evaluated with the relevant kits. The apoptosis of ox-LDL-induced HAECs was determined via flow cytometry assay. The expression of hsa_circ_0003204, miR-330-5p, and nucleotide-binding oligomerization domain 2 (Nod2) was analyzed through quantitative real-time polymerase chain reaction ( $q R T-P C R)$. The relationship between hsa_circ_0003204 or Nod2 and miR-330-5p was verified by dual-luciferase reporter assay. Protein level of Nod 2 was detected using western blot analysis.

Results: Hsa_circ_0003204 and Nod2 were upregulated while miR-330-5p was decreased in oxLDL-induced HAECs. Hsa_circ_0003204 depletion restrained the oxidative stress and apoptosis of ox-LDL-induced HAECs. Notably, hsa_circ_0003204 regulated Nod2 expression via sponging miR330-5p in HAECs. Moreover, miR-330-5p inhibition restored the constraint of the oxidative stress and apoptosis of ox-LDL-induced HAECs caused by hsa_circ_0003204 silencing. Additionally, miR-330-5p targeted Nod 2 and Nod 2 enhancement abolished the repressive effects of miR-330-5p overexpression on the oxidative stress and apoptosis of ox-LDL-induced HAECs.

Conclusions: Hsa_circ_0003204 exhaustion mitigated endothelial cell injury through suppressing the oxidative stress and apoptosis in ox-LDL-induced HAECs via the miR-330-5p/Nod2 axis.
\end{abstract}

Key words: hsa_circ_0003204, miR-330-5p,Nod2, ox-LDL, AS.

(Cent Eur J Immunol 2021; 46 (2): 140-151)

\section{Introduction}

Atherosclerosis (AS) is a common vascular disease characterized by lipid and fibrous elements amassing in the wall of the artery [1]. The pathogenesis of AS is connected with elevated oxidative stress, expression of chemokines/ cytokines, and activation of pro-inflammatory signaling pathways [2]. Also, a crucial factor in the progression of AS is oxidized low-density lipoprotein (ox-LDL) [3]. OxLDL participated in AS mainly by inducing apoptosis and oxidative stress of endothelial cells and destroying the antioxidant and secretory activity of the vascular endothelium [4]. Consequently, it is indispensable to probe into the potential molecular mechanisms of AS to provide possible strategies for preventing or treating AS.

Circular RNAs (circRNAs) are a type of covalent cyclic endogenous RNAs that are formed by the covalent attachment to the $5^{\prime}$ splice acceptor in the reverse order by a 3' splice donor of pre-mRNA [5]. A few possible fea-

Correspondence: Wei Yan, Department of Vasculocardiology, People's Hospital of Tongchuan, No. 5 Jiankang Road, Wangyi District, Tongchuan, Shaanxi, 727000, China, phone: +86-0919-2158913, e-mail: hutcth@163.com Submitted: 6.01.2020; Accepted: 30.04.2020 
tures have been reported: binging protein, miRNA sponge, and translation regulators [6]. Moreover, circRNA was revealed to be connected with many diseases, including AS [7]. For example, circular RNA hsa_circ_0044073 expedited cell proliferation and invasion in AS [8]. Circular RNA hsa_circ_0003204 (hsa_circ_0003204) originated from chromosomal region $17 \mathrm{q} 25.3$ and was an exon transcript of ubiquitin-specific peptidase 36 (USP36). Hsa_circ_0003204 was found to be enhanced in human umbilical vein endothelial cells treated with ox-LDL [9]. However, the role of hsa_circ_0003204 and its molecular mechanism in AS are unclear.

MicroRNAs (miRNAs) are an excellent class of non-coding RNAs (approximately 22 nucleotides) that exert their functions primarily via translational inhibition or mRNA degradation for the post-transcriptional regulation of gene expression [10]. MicroRNA-330-5p (miR-330-5p) has been revealed to be associated with AS progression [11], carotid plaque's stability [12] and tumor occurrence $[13,14]$. Nevertheless, whether miR-330-5p is regulated by hsa_circ_0003204 in the progression of AS has not been reported.

The nucleotide-binding oligomerization domain 2 (Nod2), which is rich in leucine repeats, is a member of the nucleotide-binding and oligomerization domain (NOD)-like receptor (NLR) family [15]. It is a major recognition receptor that integrates inflammation and endoplasmic reticulum stress and plays crucial roles in tissue homeostasis and innate antibacterial immunity [16]. It was claimed that Nod2 plays vital roles in the inflammatory process of AS [17]. Also, Nod2 was revealed to be connected with endothelial cell reactive oxygen species (ROS) generation [18] and injury [19, 20]. However, the molecular mechanisms associated with Nod2 in AS have rarely been reported.

Hence, we investigated the effect of ox-LDL on the viability of human primary aortic endothelial cells (HAECs) and constructed an AS cell model. Moreover, the expression pattern and role of hsa_circ_0003204 in the AS cell model were probed. Furthermore, the mechanism of the hsa_circ_0003204/miR-330-5p/Nod2 axis in HAEC cells was investigated, providing possible tactics for the prevention or treatment of AS.

\section{Material and methods}

\section{Cell culture}

Human primary aortic endothelial cells (HAECs) were procured from American Tissue Culture Collection (Manassas, VA, USA). An incubator in a humidifier with $5 \% \mathrm{CO}_{2}$ at $37^{\circ} \mathrm{C}$ was utilized to cultivate HAECs. Roswell Park Memorial Institute (RPMI) 1640 medium (HyClone, Logan, UT, USA) supplemented with fetal bovine serum (10\%, FBS, Atlanta Biologicals, Atlanta, GA) was applied to culture HAECs. Synchronously, streptomycin (1\%, Bao- manbio, Shanghai, China) and penicillin (1\%, Baomanbio) were replenished to the RPMI 1640 medium.

\section{Cell treatment}

HAECs were cultured in RPMI 1640 medium with different concentrations of ox-LDL $(10,20,40 \mu \mathrm{g} / \mathrm{ml})$ for $24 \mathrm{~h}$ in order to evaluate the effect of ox-LDL on the viability of HAECs. Also, HAECs were cultured in RPMI 1640 medium with ox-LDL ( $20 \mu \mathrm{g} / \mathrm{ml})$ for $24 \mathrm{~h}, 48 \mathrm{~h}$, and $72 \mathrm{~h}$ to assess the effect of ox-LDL on HAEC viability. HAECs with ox-LDL $(20 \mu \mathrm{g} / \mathrm{ml})$ treatment for $48 \mathrm{~h}$ were used as an AS cell model for further studies.

\section{Cell transfection}

Small interfering RNA targeting hsa_circ_0003204 (si-hsa_circ_0003204-1 and si-hsa_circ_0003204-2) and negative control (si-NC) were obtained from Genepharma (Shanghai, China). The miRNA mimic or inhibitor targeting miR-330-5p (miR-330-5p or in-miR-330-5p) and their corresponding negative control (miR-NC or in-miR-NC) were obtained from Ambion Inc. (Austin, TX, USA). The pcDNA3.1 vector (Invitrogen, Carlsbad, CA, USA) was applied to establish the pcDNA-Nod2 overexpression vector (Nod2). Lipofectamine 3000 reagent (Invitrogen) was utilized for the transfection of ox-LDL-induced HAECs.

\section{Cell viability}

The viability of HAECs with or without ox-LDL treatment was determined counting kit-8 (CCK8) assay. In short, HAECs $\left(2 \times 10^{4}\right)$ were maintained in medium with different concentrations of ox-LDL $(10,20,40 \mu \mathrm{g} / \mathrm{ml})$ for $24 \mathrm{~h}$ or were maintained in medium with ox-LDL $(20 \mu \mathrm{g} / \mathrm{ml})$ for $24 \mathrm{~h}, 48 \mathrm{~h}$, and $72 \mathrm{~h}$. Then, CCK8 (10 $\mu \mathrm{l}$, Dojindo Laboratories, Tokyo, Japan) was replenished to each well for $2 \mathrm{~h}$. Following this, the color reaction at $450 \mathrm{~nm}$ was determined through the Microplate Absorbance Reader (Thermo Fisher Scientific, Waltham, MA, USA).

\section{Reactive oxygen species, malondialdehyde and superoxide dismutase measurement}

The Cellular Reactive Oxygen Species Detection Assay Kit (Abcam, Cambridge, MA, USA) and Infinite 200 PRO Microplate Reader (Sunrise, Tecan, Switzerland) were used for detection of the level of ROS in the supernatant of ox-LDL-induced HAECs with or without transfection. The Lipid Peroxidation Assay Kit (Abcam) was utilized for evaluation of the level of malondialdehyde (MDA) in the supernatant of ox-LDL-induced HAECs with or without transfection, and the Infinite $200 \mathrm{PRO}$ Microplate Reader was used to determine the color reaction at $532 \mathrm{~nm}$. The Superoxide Dismutase Activity Assay Kit (Abcam) was applied to analyze the level of superoxide dismutase (SOD) in the supernatant of ox-LDL-induced 
HAECs with or without transfection, and the color reaction at $450 \mathrm{~nm}$ was determined by the Infinite 200 PRO Microplate Reader.

\section{Flow cytometry assay}

The apoptosis rate of ox-LDL-induced HAECs was assessed through the Annexin V-fluorescein isothiocyanate (FITC)/propidium iodide (PI) apoptosis detection kit (BD Biosciences, San Jose, CA, USA). Briefly, ox-LDLinduced HAECs with or without transfection were cultured in the medium for $48 \mathrm{~h}$. After washing, the cells were re-suspended in binding buffer $(200 \mu \mathrm{l})$ at a concentration of $2 \times 10^{5}$ cells $/ \mathrm{ml}$. Following this, the cells were stained with Annexin V-FITC $(5 \mu \mathrm{l})$ and PI $(100 \mu \mathrm{l})$ for $30 \mathrm{~min}$ in the dark. Eventually, the apoptosis rate of ox-LDL-induced HAECs with or without transfection was assessed through the FACScan flow cytometry (BD Biosciences).

\section{Quantitative real-time polymerase chain reaction (qRT-PCR)}

Total RNA of HAECs and ox-LDL-induced HAECs with or without transfection was extracted through TRIzol reagent (Thermo Fisher Scientific). Moloney Murine Leukemia Virus (M-MLV) First Strand Kit (Life Technologies, Grand Island, NY, USA) or One Step PrimeScript miRNA cDNA Synthesis Kit (Takara, Dalian, China) was utilized for generation of the first-strand complementary DNA of hsa_circ_0003204, Nod2, and miR-330-5p. The SYBR Fast qPCR Mix (Thermo Fisher Scientific) was used to analyze the levels of hsa_circ_0003204, miR-330-5p and Nod2. U6 small nuclear RNA (snRNA) or glyceraldehyde-3-phosphate dehydrogenase (GAPDH) was acted as an internal control for miR-330-5p, hsa_circ_0003204, and Nod2. The levels of hsa_circ_0003204, miR-330-5p, and Nod 2 were calculated by the $2^{-\Delta \Delta C t}$ method. Primers for hsa_circ_0003204 were purchased from Sangon Biotech (Shanghai, China). The primers used were as follows: miR-330-5p: 5'-TCTCTGGGCCTGTGTCTTAGGC-3' (F) and 5'-TTAATGGGGTGATTGGTGGT-3' (R); Nod2: 5'-CCAGCGTCTTTGGCCATTCAACAT-3' (F) and 5'-TTGAGCTCATCCAGTGCTTGGAGT-3' (R); GAPDH: 5'-GACTCCACTCACGGCAAATTCA-3' (F) and 5'-TCGCTCCTGGAAGATGGTGAT-3' (R), Lectin-like oxLDL receptor-1 (LOX-1): 5'-TTACTCTCCATGGTGGTGCC-3' (F) and 5'- AGCTTCTTCTDCTTGTTGCC-3' (R) as well as U6 snRNA: 5'-GCTCGCTTCGGCAGCACA-3' (F) and 5'-GAGGTATTCGCACCAGAGGA-3'(R).

\section{Dual-luciferase reporter assay}

The CircInteractome database or DIANA microT-CDS database was employed for prediction of the binding sites between hsa_circ_0003204 or Nod 2 and miR-330-5p. The sequences of hsa_circ_0003204 and Nod2 3' untranslated regions (UTR) (with predicted miR-330-5p binding sites) or their mutants were amplified and embedded into the pGL3 vector (Promega, Fitchburg, WI, USA) for construction of the luciferase reporter vector of wild-type hsa_circ_0003204 (hsa_circ_0003204 WT) and wild-type Nod2 3'UTR (Nod2 3'UTR WT) as well as mutant hsa_ circ_0003204 (hsa_circ_0003204 MUT) and mutant Nod 2 3'UTR (Nod2 3'UTR MUT). Next, the luciferase reporter vectors were transfected into HAECs with miR-330-5p, miR-NC, in-miR-330-5p, or in-miR-NC using Lipofectamine 3000 transfection reagent (Invitrogen). In the end, the dual-luciferase reporter assay kit (Promega) was used to evaluate the luciferase activities of the luciferase reporter vectors in HAECs.

\section{Western blot analysis}

Total protein of ox-LDL-induced HAECs with transfection was extracted with radio-immunoprecipitation assay (RIPA) lysis buffer (Thermo Fisher Scientific). Next, total protein was segregated with sodium dodecyl sulfate-polyacrylamide gel electrophoresis (8-12\%, SDS-PAGE). Following that, the wet electrophoretic transfer method was executed for transference of the segregated protein to polyvinylidene difluoride (PVDF) membranes (Millipore, Billerica, MA, USA). Afterward, tris buffered saline tween (TBST) buffer with 5\% skim milk was employed to block the PVDF membranes. After that, the PVDF membranes were incubated with primary antibodies overnight at $4{ }^{\circ} \mathrm{C}$. The antibodies were as follows: anti-Nod2 (ab31488, $1:$ 1000, Abcam), anti-LOX-1 (ab60178, $1: 1000$, Abcam), and anti- $\beta$-actin (ab11003, $1: 1000$, Abcam). Then, the membranes were incubated with goat anti-mouse (ab205719, $1: 2000$, Abcam) or rabbit (ab205718, $1: 2000$, Abcam) IgG. $\beta$-actin was regarded as a loading control. Finally, the bands were visualized with the Immobilon Western Chemiluminescent HRP Substrate (Millipore).

\section{Statistical analysis}

The data in the study were derived from at least 3 independent experiments. GraphPad Prism 6.0 (GraphPad, San Diego, CA, USA) and SPSS 20.0 software (IBM, Armonk, NY, USA) were used for statistical analysis. The differences between two groups were analyzed through Student's $t$ test. Data are shown as mean \pm standard deviation. Differences with $p<0.05$ were statistically significant.

\section{Results}

\section{Hsa_circ_0003204 was elevated in ox-LDL-induced HAECs}

In the first place, RPMI 1640 medium with different concentrations of ox-LDL $(10,20,40 \mu \mathrm{g} / \mathrm{ml})$ was utilized to test the effect of ox-LDL on the viability of HAECs. Results of CCK-8 showed that the viability of HAECs induced by ox-LDL was evidently repressed in a concentra- 

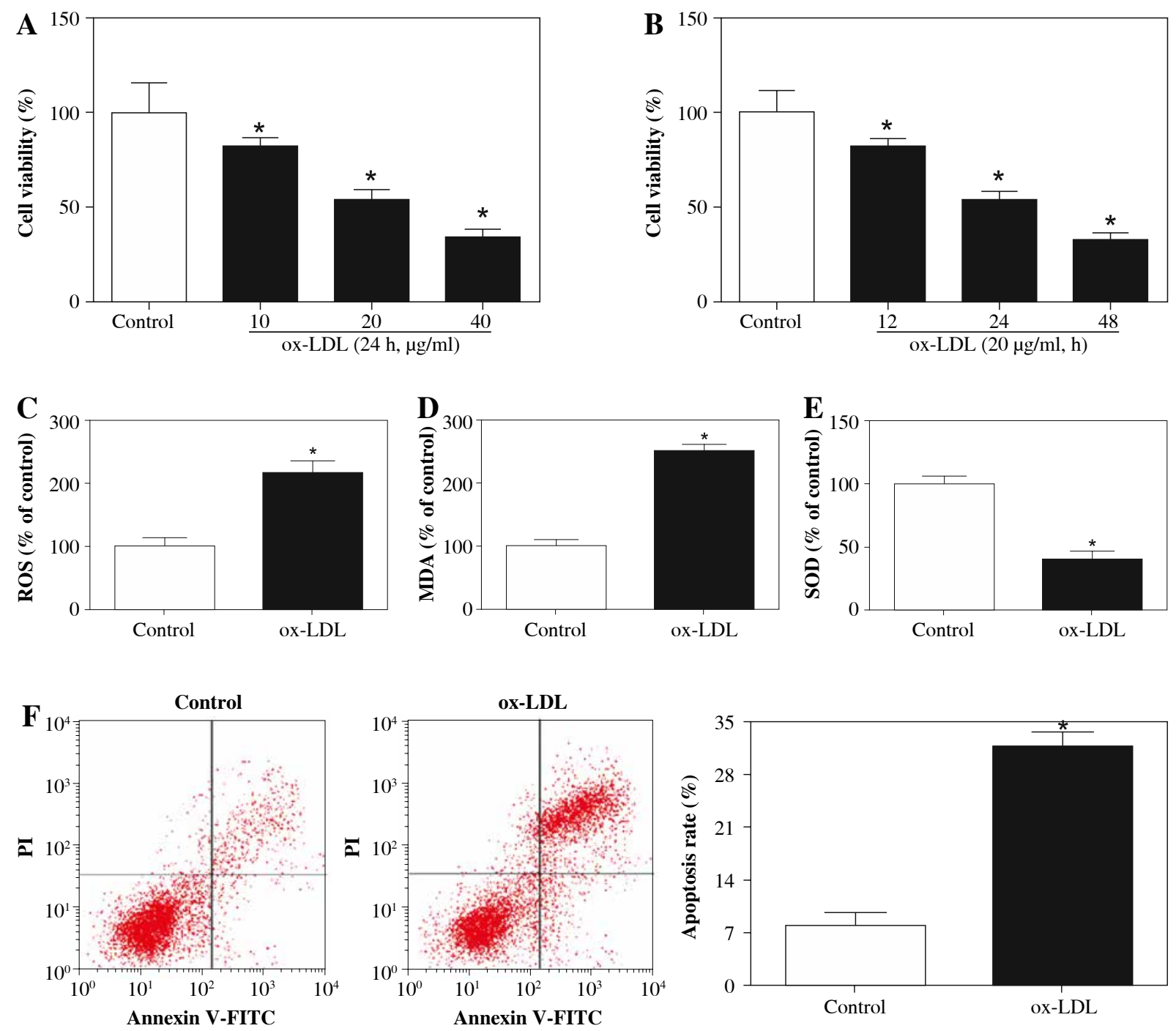

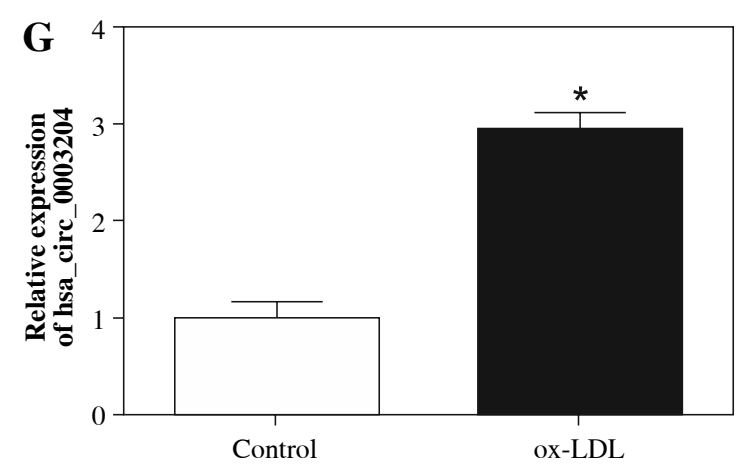

tion-dependent manner compared with the control group (Fig. 1A). Moreover, the viability of HAECs treated with ox-LDL $(20 \mu \mathrm{g} / \mathrm{ml})$ for $12 \mathrm{~h}, 24 \mathrm{~h}, 48 \mathrm{~h}$ was determined with CCK-8 assay. The results showed that the viability of HAECs treated with ox-LDL was dramatically constrained
Fig. 1. Effect of ox-LDL on expression of hsa_circ 0003204 in HAECs. A) CCK-8 assay was conducted to determine the viability of HAECs treated with ox-LDL $(10,20,40 \mu \mathrm{g} / \mathrm{ml})$ for $24 \mathrm{~h}$. B) CCK-8 assay was carried out for assessment of the viability of HAECs treated with oxLDL ( $20 \mu \mathrm{g} / \mathrm{ml})$ for $12 \mathrm{~h}, 24 \mathrm{~h}, 48 \mathrm{~h}$. C-E) Levels of ROS, MDA and SOD in the supernatant of ox-LDL-induced HAECs were analyzed with the microplate reader. F) Flow cytometry assay was employed to detect apoptosis of oxLDL-induced HAECs. G) qRT-PCR was applied to assess the expression of hsa_circ_0003204 in ox-LDL-induced HAECs. Data are shown as mean \pm standard deviation of 3 independent experiments. $* p<0.05$

in a time-dependent manner (Fig. 1B). Also, HAECs were induced with ox-LDL $(20 \mu \mathrm{g} / \mathrm{ml})$ for $48 \mathrm{~h}$ for subsequent studies. Next, we assessed the oxidative stress and apoptosis of ox-LDL-induced HAECs. The results showed that the levels of ROS and MDA in the supernatant of 


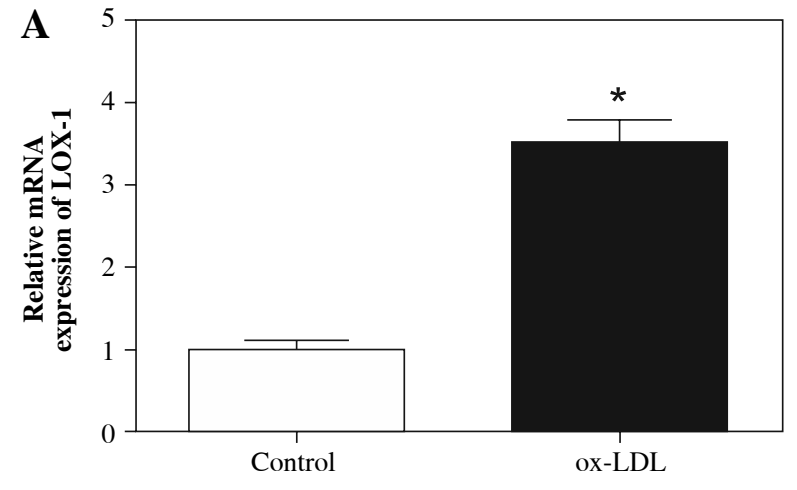

Fig. 2. Expression level of LOX-1 in ox-LDL-induced HAECs. A, B) Levels of LOX-1 mRNA and protein in ox-LDL-induced HAECs were evaluated through qRTPCR or western blot analysis. Data are shown as mean \pm standard deviation of 3 independent experiments. ${ }^{*} p<0.05$

ox-LDL-induced HAECs were elevated, but the level of SOD was impeded (Fig. 1C-E). Flow cytometry assay revealed that the apoptosis rate of ox-LDL-induced HAECs was remarkably higher than that in the control group (Fig. 1F). LOX-1 is a receptor for ox-LDL, which regulates the biological function of ox-LDL. We observed that the levels of LOX-1 mRNA and protein were elevated in ox-LDL-induced HAECs (Fig. 2A and B). Therefore, the induction of HAECs with ox-LDL $(20 \mu \mathrm{g} / \mathrm{ml})$ for $48 \mathrm{~h}$ was used as an AS cell model for further study. Subsequently, the expression of hsa_circ_0003204 in ox-LDL-induced HAECs was evaluated by qRT-PCR. As exhibited in Figure 1G, the level of hsa_circ_0003204 was apparently higher in ox-LDL-induced HAECs compared to the control group. In sum, high hsa_circ_0003204 expression might be connected with the process of AS.

\section{Hsa_circ_0003204 depletion constrained the oxidative stress and apoptosis of ox-LDL-induced HAECs}

Taking into account the above results, we further probed into the role of hsa_circ_0003204 in the process of AS by loss-of-function experiments. In the first place, the si-NC, si-hsa_circ_0003204-1, or si-hsa_circ_0003204-2 was transfected into ox-LDL-induced HAECs to silence the expression of hsa_circ_0003204. Results of qRT-PCR indicated that hsa_circ_0003204 was obviously reduced in ox-LDL-induced HAECs transfected with si-hsa_circ_0003204-1 and si-hsa_circ_0003204-2 in contrast to the si-NC group (Fig. 3A). Afterward, the effects of hsa_circ_0003204 inhibition on the oxidative stress and apoptosis of ox-LDL-induced HAECs were explored. The results demonstrated that ROS and MDA were conspicuously suppressed, while SOD was distinctly accelerated in ox-LDL-induced HAECs transfected
B
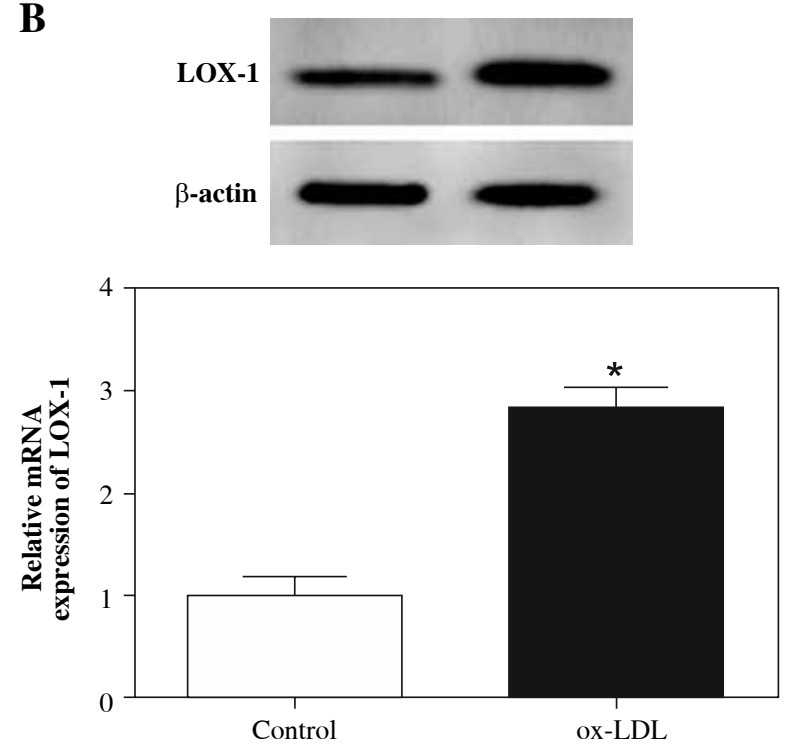

with si-hsa_circ_0003204-1 and si-hsa_circ_0003204-2 compared with the control group (Fig. 3B-D). Also, flow cytometry revealed that si-hsa_circ_0003204-1 and si-hsa_ circ_0003204-2 could drastically impede the apoptosis of ox-LDL-induced HAECs vs. the control si-NC (Fig. 3E). Taken together, these results suggested that inhibition of hsa_circ_0003204 could attenuate ox-LDL induced injury of HAECs by repressing oxidative stress and apoptosis.

\section{Hsa_circ_0003204 acted as a sponge of miR-330-5p}

To further understand how hsa_circ_0003204 played its function in AS, the CircInteractome database was used for the prediction of the target of hsa_circ_0003204. We discovered that miR-330-5p possessed potential binding sites for hsa_circ_0003204 (Fig. 4A). Afterward, we constructed luciferase reporter vectors with hsa_circ_0003204 WT and hsa_circ_0003204 MUT to execute the dual-luciferase reporter assay for the verification of binding sites between hsa_circ_0003204 and miR-330-5p. The results showed that the luciferase activity of the luciferase reporter vectors containing hsa_circ_0003204 WT was strikingly lower in HAECs transfected with miR-330-5p than the miR-NC group (Fig. 4B). Moreover, silenced miR-330-5p expression enhanced the luciferase activity of the luciferase reporter vectors containing hsa_circ_0003204 WT in HAECs compared to the vector control group (Fig. 4C). Additionally, qRT-PCR was conducted to detect miR-330-5p expression in ox-LDL-induced HAECs. The results showed that miR-330-5p was dramatically downregulated in ox-LDL-induced HAECs (Fig. 4D). Also, a conspicuous augmentation of miR-330-5p was observed in ox-LDL-induced HAECs transfected with si-hsa_circ_0003204-1 and si-hsa_circ_0003204-2 (Fig. 4E). In short, these findings demonstrated that hsa_circ_0003204 served as a sponge of miR-330-5p in ox-LDL-induced HAECs. 

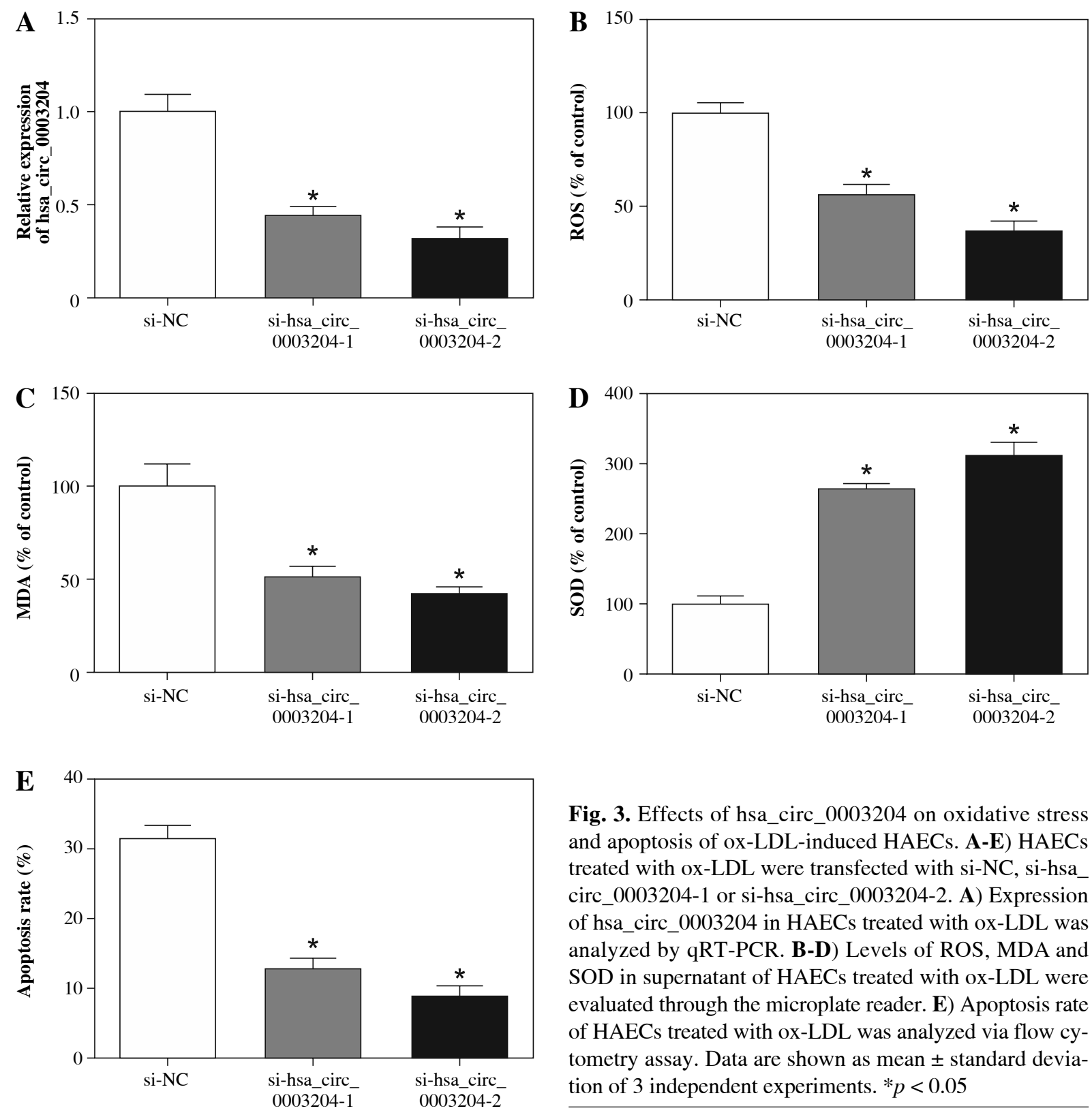

Fig. 3. Effects of hsa_circ_0003204 on oxidative stress and apoptosis of ox-LDL-induced HAECs. A-E) HAECs treated with ox-LDL were transfected with si-NC, si-hsa_ circ_0003204-1 or si-hsa_circ_0003204-2. A) Expression of hsa_circ_0003204 in HAECs treated with ox-LDL was analyzed by qRT-PCR. B-D) Levels of ROS, MDA and SOD in supernatant of HAECs treated with ox-LDL were evaluated through the microplate reader. E) Apoptosis rate of HAECs treated with ox-LDL was analyzed via flow cytometry assay. Data are shown as mean \pm standard deviation of 3 independent experiments. $* p<0.05$

MiR-330-5p silencing reversed hsa_circ_0003204 knockdown-mediated effects on cell oxidative stress and apoptosis in ox-LDL-induced HAECs

In view of the above results, we further explored whether hsa_circ_0003204 mediated the oxidative stress and apoptosis of ox-LDL-induced HAECs via miR-330-5p. At the outset, qRT-PCR was carried out to detect the expression of miR-330-5p in ox-LDL-induced HAECs transfected with si-NC, si-hsa_circ_0003204-2, si-hsa circ_0003204-2 + in-miR-NC, or si-hsa_circ_0003204-2 + in-miR-330-5p. The results indicated that the enhancement of miR-330-5p in ox-LDL-induced HAECs caused

by hsa_circ_0003204 knockdown was abolished by the inhibition of miR-330-5p (Fig. 5A). Following this, the effects of miR-330-5p suppression on the oxidative stress and apoptosis of ox-LDL-induced HAECs transfected with si-hsa_circ_0003204-2 were further evaluated. The results showed that both the reduction of ROS and MDA and the augmentation of SOD in ox-LDL-induced HAECs caused by hsa_circ_0003204 downregulation were overturned by the inhibition of miR-330-5p (Fig. 5B-D). Flow cytometry indicated that decreased miR-330-5p expression could restore hsa_circ_0003204 repression-mediated effects on cell oxidative stress and apoptosis in ox-LDL-induced HAECs (Fig. 5E). Therefore, these results demonstrated that hsa_ 
A $\begin{array}{ll}\text { hsa_circ_0003204 WT } & \text { 5'...GGCCAGUGAGCCCCCCCAGAGC...3' } \\ \text { hsa-miR-330-5p } & \text { 3' }{ }^{\prime} \text { CGGAUUCUGUGUCCGGGUCUCU 5' } \\ \text { hsa_circ_0003204 MUT } & \text { 5'...GGCCAGUGAGCCCCGGGUCUCC...3' }\end{array}$
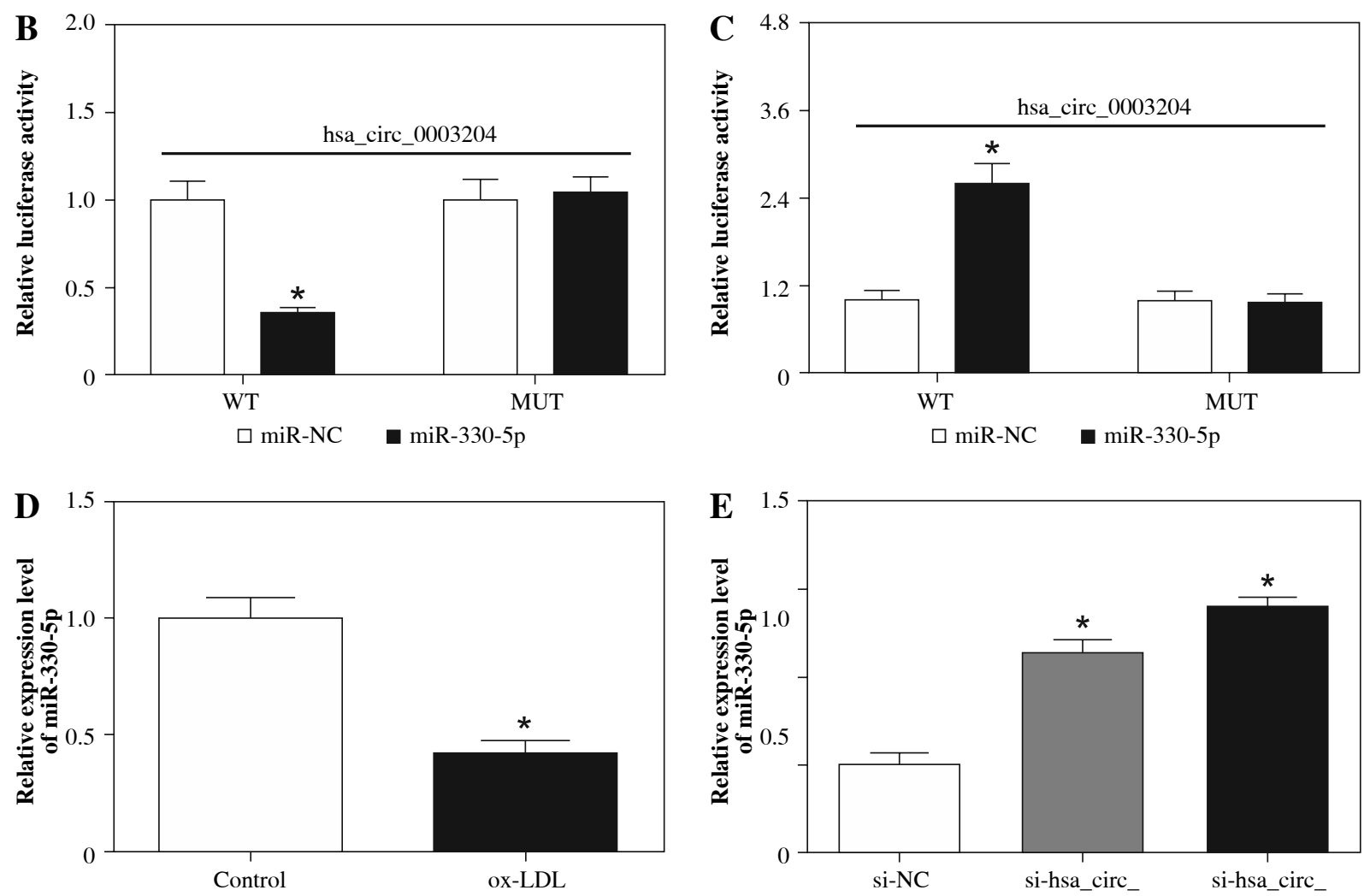

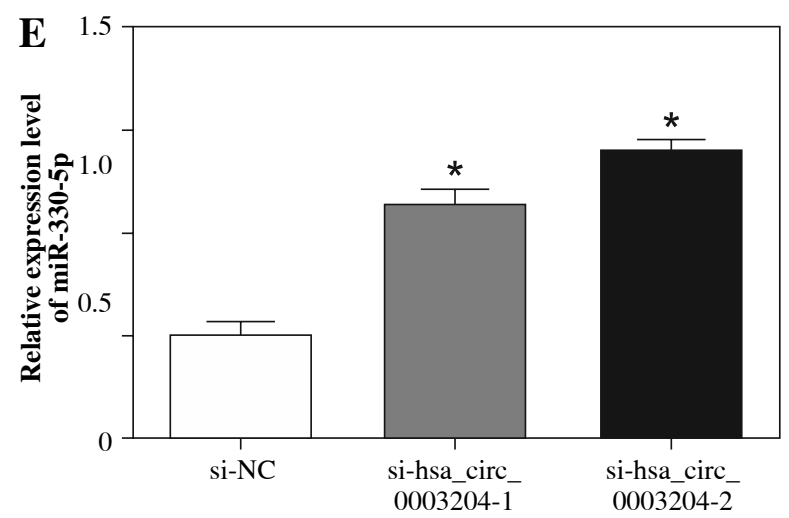

Fig. 4. MiR-330-5p was a target of hsa_circ_0003204 in HAECs. A) Binding sites between miR-330-5p and hsa_ circ_0003204 were predicted by the CircInteractome database. B, C) Activities of luciferase reporter vectors (hsa_ circ_0003204 WT and hsa_circ_0003204 MUT) in HAECs transfected with miR-NC (B), miR-330-5p (B), in-miR-NC, (C) or in-miR-330-5p (C) were evaluated with dual-luciferase reporter assay. D) Expression of miR-330-5p in ox-LDLinduced HAECs was detected by qRT-PCR. E) Level of miR-330-5p in ox-LDL-induced HAECs transfected with siNC, si-hsa_circ_0003204-1, or si-hsa_circ_0003204-2 was analyzed via qRT-PCR. Data are shown as mean \pm standard deviation of 3 independent experiments. ${ }^{*} p<0.05$

circ_0003204 regulated cell oxidative stress and apoptosis in ox-LDL-induced HAECs through miR-330-5p.

\section{Nod2 was verified to act as a target of miR-330-5p in HAECs}

To figure out how miR-330-5p exerted its role in AS, the DIANA microT-CDS database was employed to predict the target of miR-330-5p in HAECs. As presented in Figure 6A, Nod2 was predicted to have possible binding sites for miR-330-5p. Then, the luciferase reporter vectors with Nod2 3'UTR WT and Nod2 3'UTR MUT were established. The results of dual-luciferase reporter assay revealed that miR-330-5p specially reduced the activity of the luciferase reporter vectors containing Nod2 3'UTR WT in HAECs, while the activity of the luciferase reporter vectors containing Nod2 3'UTR MUT was not apparently different (Fig. 6B). Moreover, results of qRT-PCR showed that the mRNA level of Nod2 was remarkably boosted in ox-LDL-induced HAECs (Fig. 6C). Also, the protein level of Nod2 in ox-LDL-induced HAECs transfected with miR-NC, miR-330-5p, in-miR-NC, or in-miR-330-5p was analyzed with western blot analysis. As expected, 

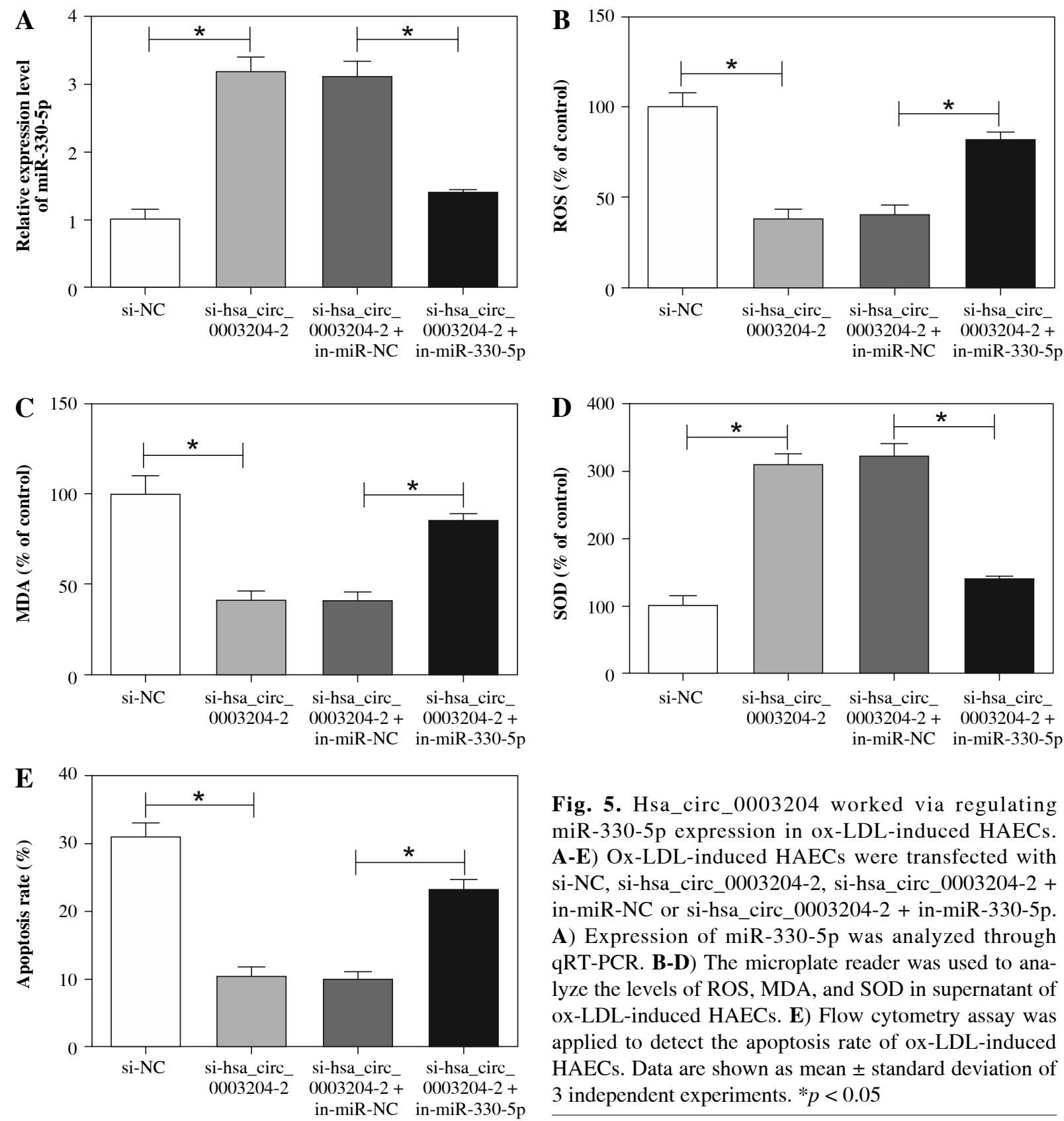

miR-330-5p overexpression dramatically decreased the protein level of Nod2 in ox-LDL-induced HAECs, whereas downregulation of miR-330-5p drastically elevated the Nod 2 protein level, indicating that Nod 2 was negatively regulated by miR-330-5p (Fig. 6D). Furthermore, we discovered that Nod 2 protein level was evidently downregulated in ox-LDL-induced HAECs transfected with si-hsa_ circ_0003204-2, while this reduction was recovered by the introduction of in-miR-330-5p, indicating that Nod2 was regulated by hsa_circ_0003204 and miR-330-5p (Fig. 6E). Collectively, these results suggested that Nod2 was regulated by hsa_circ_0003204 via miR-330-5p in HAECs.

Fig. 5. Hsa_circ_0003204 worked via regulating miR-330-5p expression in ox-LDL-induced HAECs. A-E) Ox-LDL-induced HAECs were transfected with si-NC, si-hsa_circ_0003204-2, si-hsa_circ_0003204-2 + in-miR-NC or si-hsa_circ_0003204-2 + in-miR-330-5p. A) Expression of miR-330-5p was analyzed through qRT-PCR. B-D) The microplate reader was used to analyze the levels of ROS, MDA, and SOD in supernatant of ox-LDL-induced HAECs. E) Flow cytometry assay was applied to detect the apoptosis rate of ox-LDL-induced HAECs. Data are shown as mean \pm standard deviation of 3 independent experiments. $* p<0.05$

\section{Nod2 augmentation reversed the effects of miR-330-5p on the oxidative stress and apoptosis of ox-LDL-induced HAECs}

Knowing that miR-330-5p negatively regulated Nod2 expression, we next investigated whether miR-330-5p mediated the oxidative stress and apoptosis of ox-LDL-induced HAECs via Nod2. Firstly, the protein level of Nod2 in ox-LDL-induced HAECs transfected with miR-NC, miR-330-5p, miR-330-5p + pcDNA, or miR-330-5p + Nod 2 was assessed with western blot analysis. The results indicated that the inhibition of Nod 2 protein mediated by 
A $\begin{array}{ll}\text { Nod2 3'UTR WT } & 5, \text {...ACUCCAGGGCCAAGCCCAGAGC...3' } \\ \text { hsa-miR-330-5p } & \text { 3' } \text { CGGAUUCUGUGUCCGGGUCUCU 5' } \\ \text { Nod2 3'UTR MUT } & 5 \text { '...ACUCCAGGGCCAACGGGUCUCC...3', }\end{array}$
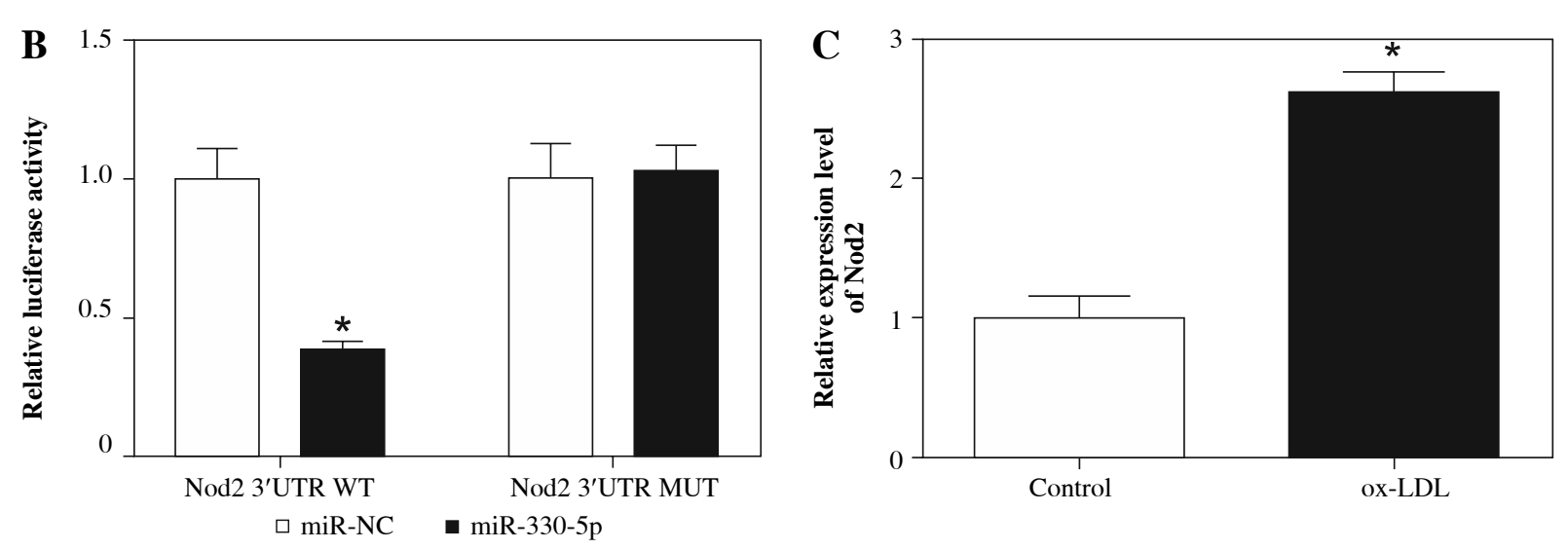

D

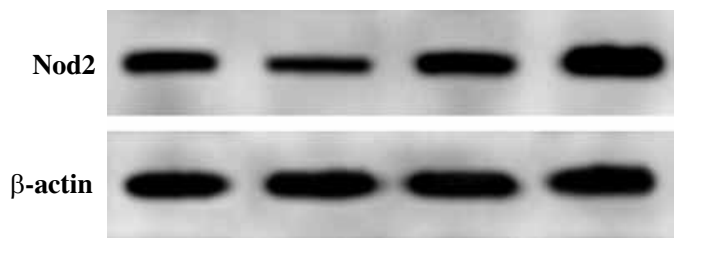

E
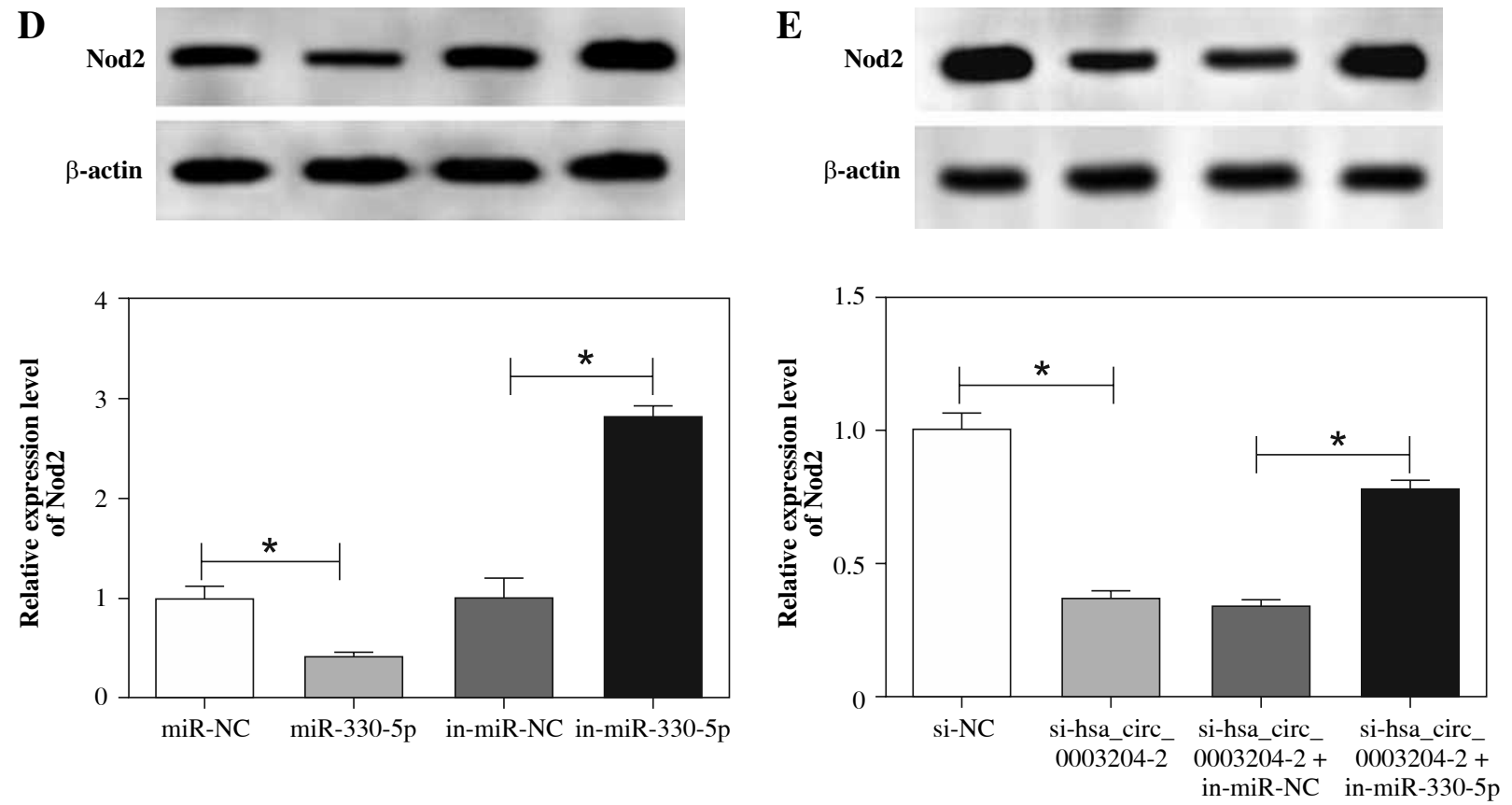

Fig. 6. MiR-330-5p targeted Nod 2 in HAECs. A) Binding sites of miR-330-5p in Nod 2 were predicted by the DIANA microT-CDS database. B) Luciferase activities of Nod2 3'UTR WT and Nod2 3'UTR MUT in HAECs transfected with miR-330-5p or miR-NC were assessed by dual-luciferase reporter assay. C) mRNA level of Nod2 in ox-LDL-induced HAECs was measured through qRT-PCR. D) Protein level of Nod2 in ox-LDL-induced HAECs transfected with miR-NC, miR-330-5p, in-miR-NC, or in-miR-330-5p was evaluated using western blot analysis. E) Western blot analysis was carried out for evaluation of the protein level of Nod2 in ox-LDL-induced HAECs transfected with si-NC, sihsa_circ_0003204-2, si-hsa_circ_0003204-2 + in-miR-NC, or si-hsa_circ_0003204-2 + in-miR-330-5p. Data are shown as mean \pm standard deviation of 3 independent experiments. $* p<0.05$ 
A
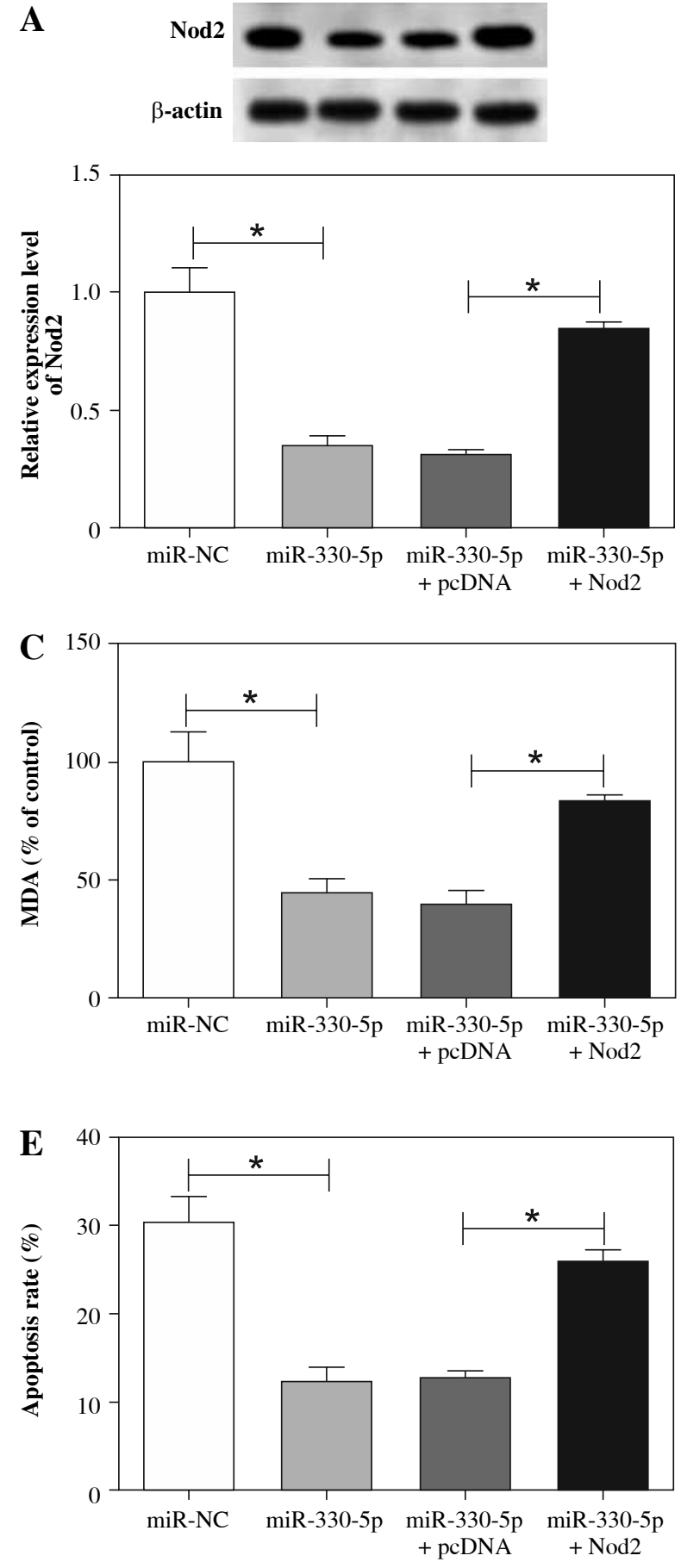

miR-330-5p upregulation was restored by the overexpression of Nod2 (Fig. 7A). Furthermore, we found that both the constraint of ROS and MDA and the acceleration of SOD in the supernatant of ox-LDL-induced HAECs prompted by miR-330-5p elevation were recovered by Nod2 upregulation (Fig. 7B-D). Moreover, Nod2 enhancement overturned the inhibition of apoptosis of ox-LDL-induced HAECs earned by miR-330-5p elevation (Fig. 7E).
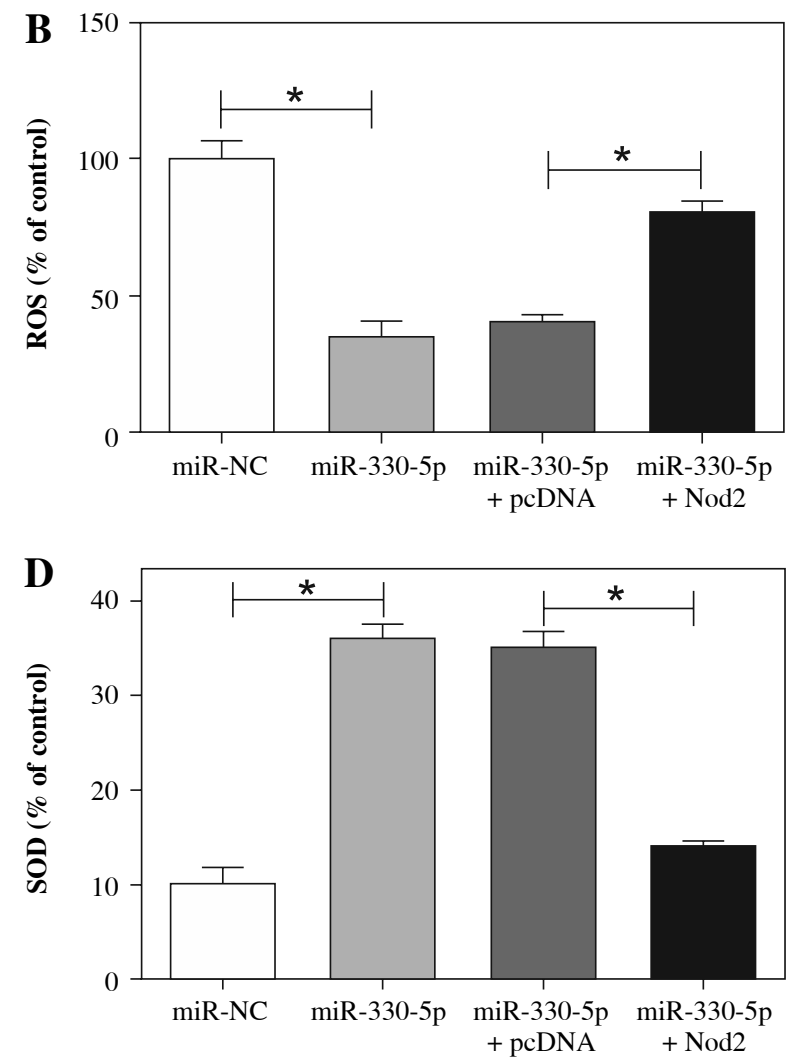

Fig. 7. MiR-330-5p regulated Nod2 expression to mediate oxidative stress and apoptosis in ox-LDL-induced HAECs. A-E) Ox-LDL-induced HAECs were transfected with miR-NC, miR-330-5p, miR-330-5p + pcDNA, or miR-330-5p + Nod2. A) Protein level of Nod2 in HAECs with ox-LDL treatment was detected by western blot analysis. B-D) Levels of ROS, MDA, and SOD in the supernatant of HAECs with ox-LDL treatment were analyzed with a microplate reader. E) Apoptosis rate of HAECs with ox-LDL treatment was determined through flow cytometry assay. Data are shown as mean \pm standard deviation of 3 independent experiments. $* p<0.05$

Together, these data indicated that miR-330-5p modulated cell oxidative stress and apoptosis in ox-LDL-induced HAECs by Nod2.

\section{Discussion}

Atherosclerosis is a major health problem for the elderly worldwide, and it can cause cardiovascular diseases 


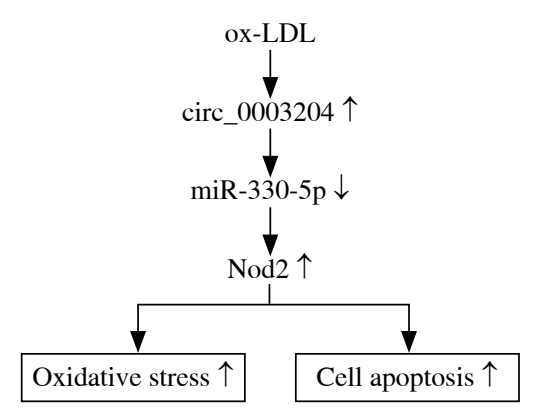

Fig. 8. Schematic diagram of the mechanism of hsa circ_0003204 induced by ox-LDL involved in endothelial cell injury

such as stroke and coronary heart disease [21]. Studies have shown that circRNA is stable with cell type-specific or tissue-specific expression, and it is a promising disease biomarker [22]. Hsa_circ_0003204 was claimed to be augmented in vascular endothelial cells with ox-LDL treatment, while the role of hsa_circ_0003204 in AS was unclear.

Oxidative stress plays vital roles in AS pathogenesis by participating in the oxidative modification of ox-LDL. Its imbalance reduced the body's inherent antioxidant defense system or contributed to the production of ROS [23]. Also, an excess of ROS could cause cardiovascular disease [24]. SOD is a vascular wall antioxidant enzyme that converts superoxide to hydrogen peroxide [25]. Elevated serum MDA levels might play a vital role in the development of AS, leading to coronary heart disease [26]. In this study, HAECs were treated with ox-LDL to construct AS models. We discovered that ROS and MDA levels were enhanced in the supernatant of ox-LDL-induced HAECs, while the SOD level was reduced. Moreover, the apoptosis was promoted and the expression of hsa_circ_0003204 was upregulated in HAECs with ox-LDL treatment. Furthermore, silenced hsa_circ_0003204 expression blocked the oxidative stress and apoptosis of ox-LDL-induced HAECs. Nevertheless, little is known about the expression and role of hsa_circ_0003204 in AS and the other diseases.

Studies have revealed that circRNA exerts its function by acting as a sponge of miRNA [27-29]. MiR-330-5p was claimed to be involved in the progression of AS. Wei et al. uncovered that miR-330-5p elevation might cause carotid plaque instability in patients with carotid stenosis [12]. Additionally, miR-330-5p was disclosed to be decreased in THP-1 cells with ox-LDL treatment and miR-330-5p enhancement curbed the process of oxidative stress and inflammatory THP-1 cells with ox-LDL treatment [30]. In the present study, hsa_circ_0003204 was confirmed to serve as a sponge of miR-330-5p in HAECs. Furthermore, consistent with the above studies, miR-330-5p was downregulated in ox-LDL-induced HAECs and miR-330-5p repression reversed the suppression of oxidative stress and apoptosis of ox-LDL-induced HAECs caused by hsa_ circ_0003204 knockdown.

It was reported that Nod 2 could increase lipid-rich necrotic core expression and contribute to vascular inflammation in AS [31]. Moreover, AS was associated with Nod2-mediated increase in innate immunity [16]. The report of $\mathrm{Xu}$ et al. revealed that Nod2 downregulation could mitigate endothelial cell injury induced by ox-LDL [19]. Also, the reduction of Nod2 caused by long noncoding RNA XIST inhibition could attenuate endothelial cell injury by repressing apoptosis and promoting the viability of ox-LDL-induced umbilical vein endothelial cells [20]. In this study, we discovered that Nod 2 was enhanced in ox-LDL-induced HAECs and Nod2 was the target of miR-330-5p. Moreover, the introduction of Nod2 abolished miR-330-5 upregulation-mediated oxidative stress and apoptosis of ox-LDL-induced HAECs. In addition, hsa_circ_0003204 regulated Nod2 protein via miR-330-5p in HAECs. Therefore, we verified that Nod 2 could accelerate the process of AS, in line with the previous studies. Therefore, we concluded that ox-LDL-induced hsa_ circ_0003204 regulated endothelial cell injury through the miR-330-5p/Nod2 axis (Fig. 8).

\section{Conclusions}

In sum, hsa_circ_0003204 was reinforced in ox-LDLinduced HAECs. Moreover, hsa_circ_0003204 downregulation impeded the oxidative stress and apoptosis in ox-LDL-induced HAECs through inhibiting Nod2 via miR-330-5p. Also, we reported for the first time the role of hsa_circ_0003204 and the mechanism of the hsa_ circ_0003204/miR-330-5p/Nod2 axis in AS progression. This study provided new insight for the prevention or treatment of AS.

The authors declare no conflict of interest.

\section{References}

1. Cao Y, Hui J, Kole A, et al. (2016): High-sensitivity intravascular photoacoustic imaging of lipid-laden plaque with a collinear catheter design. Sci Rep 6: 25236.

2. Kattoor AJ, Pothineni NVK, Palagiri D, et al. (2017): Oxidative stress in atherosclerosis. Curr Atheroscler Rep 19: 42.

3. Pirillo A, Norata GD, Catapano AL (2013): LOX-1, OxLDL, and atherosclerosis. Mediators Inflamm 2013: 152786.

4. Lubrano V, Balzan S (2014): LOX-1 and ROS, inseparable factors in the process of endothelial damage. Free Radic Res 48: 841-848.

5. Ashwal-Fluss R, Meyer M, Pamudurti NR, et al. (2014): circRNA biogenesis competes with pre-mRNA splicing. Mol Cell 56: 55-66.

6. Meng X, Li X, Zhang P, et al. (2017): Circular RNA: an emerging key player in RNA world. Brief Bioinform 18: 547-557. 
7. Holdt LM, Stahringer A, Sass K, et al. (2016): Circular non-coding RNA ANRIL modulates ribosomal RNA maturation and atherosclerosis in humans. Nat Commun 7: 12429.

8. Shen L, Hu Y, Lou J, et al. (2019): CircRNA0044073 is upregulated in atherosclerosis and increases the proliferation and invasion of cells by targeting miR107. Mol Med Rep 19: 3923-3932.

9. Li CY, Ma L, Yu B (2017): Circular RNA hsa_circ_0003575 regulates oxLDL induced vascular endothelial cells proliferation and angiogenesis. Biomed Pharmacother 95: 1514-1519.

10. Cai Y, Yu X, Hu S, et al. (2009): A brief review on the mechanisms of miRNA regulation. Genomics Proteomics Bioinformatics 7: 147-154.

11. Liu J, Huang GQ, Ke ZP (2019): Silence of long intergenic noncoding RNA HOTAIR ameliorates oxidative stress and inflammation response in ox-LDL-treated human macrophages by upregulating miR-330-5p. J Cell Physiol 234: 5134-5142.

12. Wei X, Sun Y, Han T, et al. (2019): Upregulation of miR-330$5 p$ is associated with carotid plaque's stability by targeting Talin-1 in symptomatic carotid stenosis patients. BMC Cardiovasc Disord 19: 149.

13. Xiao S, Yang M, Yang H, et al. (2018): miR-330-5p targets SPRY2 to promote hepatocellular carcinoma progression via MAPK/ERK signaling. Oncogenesis 7: 90.

14. Fu X, Zhang L, Dan L, et al. (2017): LncRNA EWSAT1 promotes ovarian cancer progression through targeting miR-3305p expression. Am J Transl Res 9: 4094-4103.

15. Hugot JP, Chamaillard M, Zouali H, et al. (2001): Association of NOD2 leucine-rich repeat variants with susceptibility to Crohn's disease. Nature 411: 599-603.

16. Liu HQ, Zhang XY, Edfeldt K, et al. (2013): NOD2-mediated innate immune signaling regulates the eicosanoids in atherosclerosis. Arterioscler Thromb Vasc Biol 33: 2193-2201.

17. Yuan H, Zelkha S, Burkatovskaya M, et al. (2013): Pivotal role of NOD2 in inflammatory processes affecting atherosclerosis and periodontal bone loss. Proc Natl Acad Sci U S A 110: E5059-5068.

18. Kong LJ, Liu XQ, Xue Y, et al. (2018): Muramyl dipeptide induces reactive oxygen species generation through the NOD2/COX-2/NOX4 signaling pathway in human umbilical vein endothelial cells. J Cardiovasc Pharmacol 71: 352-358.

19. Xu X, Ma C, Duan Z, et al. (2019): LncRNA ZEB1-AS1 mediates oxidative low-density lipoprotein-mediated endothelial cells injury by post-transcriptional stabilization of NOD2. Front Pharmacol 10: 397.

20. Xu X, Ma C, Liu C, et al. (2018): Knockdown of long noncoding RNA XIST alleviates oxidative low-density lipoprotein-mediated endothelial cells injury through modulation of miR-320/NOD2 axis. Biochem Biophys Res Commun 503: 586-592.

21. Mozaffarian D, Benjamin EJ, Go AS, et al. (2016): Heart disease and stroke statistics-2016 update: a report from the American Heart Association. Circulation 133: e38-360.

22. Haque S, Harries LW (2017): Circular RNAs (circRNAs) in health and disease. Genes (Basel) 8: 353.

23. Peluso I, Morabito G, Urban L, et al. (2012): Oxidative stress in atherosclerosis development: the central role of LDL and oxidative burst. Endocr Metab Immune Dis Drug Targets 12: 351-360.

24. Brieger K, Schiavone S, Miller FJ, Jr., et al. (2012): Reactive oxygen species: from health to disease. Swiss Med Wkly 142: w13659.
25. Li H, Horke S, Forstermann U (2013): Oxidative stress in vascular disease and its pharmacological prevention. Trends Pharmacol Sci 34: 313-319.

26. Khan MA, Baseer A (2000): Increased malondialdehyde levels in coronary heart disease. J Pak Med Assoc 50: 261-264.

27. Zhou B, Yu JW (2017): A novel identified circular RNA, circRNA_010567, promotes myocardial fibrosis via suppressing miR-141 by targeting TGF-beta1. Biochem Biophys Res Commun 487: 769-775.

28. Wang K, Long B, Liu F, et al. (2016): A circular RNA protects the heart from pathological hypertrophy and heart failure by targeting miR-223. Eur Heart J 37: 2602-2611.

29. Bai Y, Zhang Y, Han B, et al. (2018): Circular RNA DLGAP4 ameliorates ischemic stroke outcomes by targeting miR-143 to regulate endothelial-mesenchymal transition associated with blood-brain barrier integrity. J Neurosci 38: 32-50.

30. Liu J, Huang GQ, Ke ZP (2019): Silence of long intergenic noncoding RNA HOTAIR ameliorates oxidative stress and inflammation response in ox-LDL-treated human macrophages by upregulating miR-330-5p. BMC Cardiovasc Dis 234: 5134-5142.

31. Johansson ME, Zhang XY, Edfeldt K, et al. (2014): Innate immune receptor NOD2 promotes vascular inflammation and formation of lipid-rich necrotic cores in hypercholesterolemic mice. Eur J Immunol 44: 3081-3092. 